\title{
Evidência de Validade da Escala Brasileira de Gratidão (B-GRAT) na Psicologia Positiva
}

\author{
Ana Claudia Souza Vazquez ${ }^{1}$, Joice Franciele Friedrich Almansa \\ Universidade Federal de Ciências da Saúde, Porto Alegre-RS, Brasil \\ Clarissa Pinto Pizarro de Freitas \\ Universidade Salgado de Oliveira, Rio de Janeiro-RJ, Brasil \\ Claudio Simon Hutz \\ Universidade Federal do Rio Grande do Sul, Porto Alegre-RS, Brasil
}

\section{RESUMO}

Gratidão é um estado mental positivo de agradecimento por experiências de vida, adversas ou de risco, vinculadas à memória das lembranças afetivas que aumentam o bem-estar subjetivo. Foi desenvolvida uma escala brasileira com o objetivo de mensurar o construto da gratidão, definida para esta pesquisa. A escala original com 13 itens, construída e revisada com base nos padrões internacionais, foi preenchida por 762 participantes (625 mulheres, 82\%; 137 homens), entre 18 e 90 anos, $M=41, D P=13$. A análise fatorial exploratória extraiu sete itens com estrutura interna unidimensional, resultando na Escala Brasileira de Gratidão (B-GRAT). A análise fatorial confirmatória, pela matriz de correlações policóricas demonstrou índice adequado para o modelo testado e consistência interna de $\alpha=0,84$. Foi testada a correlação de Pearson com a Escala de Esperança Disposicional. Os resultados indicam evidência da adequação psicométrica da B-GRAT na avaliação da gratidão no Brasil.

Palavras-chave: gratidão; esperança; psicologia positiva.

\section{ABSTRACT - Evidence of validity of Brazilian Scale of Gratitude (B-GRAT), in Positive Psychology}

Gratitude is a positive mental state of gratefulness for adverse or risky life experiences related to affective memories that improve the subjective well-being. A new scale was developed aiming to produce an instrument for the assessment of gratitude. An original pool of 13 items, constructed and reviewed using accurate standards, was responded to by 762 participants (242 females, $72.5 \%$; 88 males), aged 18-90 years, $M=41, S D=13$. An Exploratory Factor Analysis concluded with the selection of 7 items that presented an internal unidimensional structure, resulting in the Brazilian scale of Gratitude (B-GRAT). Confirmatory Factor Analysis using the polychoric correlations matrix showed an adequate fit of the model and an internal consistency de $\alpha=0.84$. The concurrent validity with the Hope Index Scale was checked by means of Pearson's correlation. The results indicate evidence that the B-GRAT is psychometrically adequate to assess gratitude in Brazil.

Keywords: Gratitude, Dispositional Hope, Positive Psychology

\section{RESUMEN - Evidencia de validez de la Escala Brasileña de Gratitud (B-GRAT), en Psicología Positiva}

La gratitud es un estado mental positivo de agradecimiento por las experiencias de la vida, adversas o arriesgadas, relacionadas con la memoria afectiva que aumentan el bienestar subjetivo. Se desarrolló una escala brasileña con el propósito de medir el constructo de gratitud, definido para esta investigación. La escala original con 13 ítems, construida y revisada según los estándares internacionales, fue completada por 762 participantes (625 mujeres, $82 \%, 137$ hombres), entre 18 y 90 años, $M=41, D S=13$. El análisis factorial exploratorio reveló 7 ítems con una estructura interna unidimensional, lo que resultó en la Escala de Gratitud Brasileña (B-GRAT). El análisis factorial confirmatorio, por la matriz de correlaciones policóricas, presentó un índice adecuado para el modelo probado y una consistencia interna de $\alpha=0.84$. La correlación de Pearson fue probada con la escala de esperanza disposicional. Los resultados indican evidencia de la adecuación psicométrica de B-GRAT en la evaluación de la gratitud en Brasil.

Palabras clave: Gratitud, Esperanza, Psicología Positiva.

Gratidão é um fenômeno psicológico pesquisado na Psicologia Positiva e por autores de outras áreas, cujos achados comprovam robustamente sua relação com o bem-estar subjetivo (Bono \& McCullough, 2006; Fredrickson, 2013, 1998; Wooh, Froh, \& Gerathy, 2010;).
O desenvolvimento da gratidão ao longo da vida ou em situações de vida específicas é apontado como um fator de proteção à saúde mental. Nesse sentido, intervenções em gratidão demonstram ser benéficas para a diminuição de transtornos de depressão ou ansiedade e do sentimento de 
solidão, bem como na aplicação de estratégicas importantes ao enfrentamento de situações adversas e de risco. Além disso, as intervenções em gratidão são efetivas para contribuir na promoção do aumento de satisfação de vida, afetos positivos, extroversão e comprometimento com a saúde física (Alves, 2010; Emmons \& Mishra, 2011; Emmons \& Stern, 2013; Lyubomirsky, 2008; Pieta \& Freitas, 2009; Salvador-Ferrer, 2017; Sapienza \& Pedromônico, 2005; Watkins, Woodward, Stone \& Kolts, 2003).

Embora haja robusta evidência científica dos benefícios para o desenvolvimento humano e o bem-estar subjetivo, as teorizações sobre esse construto carecem de aprofundamento (Wood et al., 2010). Pesquisadores se dedicaram a descrever seus aspectos constitutivos, em diferentes bases epistemológicas; partindo do pressuposto comum de que a gratidão é uma emoção positiva que reflete um estado psicológico que pode ser desenvolvido ao longo da vida. Considera-se que a ampla discussão na área fundamenta a natureza ontológica do conceito de gratidão em duas funções relevantes para o comportamento humano, as quais atuam de forma concomitante: (a) social, e (b) moral (Pieta \& Freitas, 2009; Wooh et al., 2010).

A função social da gratidão é caracterizada como uma resultante da mútua troca de favores entre o beneficiário e o benfeitor, cuja relação assimétrica coloca o primeiro na posição de dependente da ação de outrem. A apreciação do favor recebido, para o qual não há possibilidade de reciprocidade simétrica no momento da ação benevolente, leva o beneficiário a vivenciar emoções positivas que o conduzem ao comportamento pró-social em suas relações (Fredrickson, 2002; McCullough et al., 2001; Schwartz, 1967). Entre os comportamentos pró-sociais apresentados pelos indivíduos que vivenciam a gratidão, identificam-se o fortalecimento dos laços positivos com outras pessoas, o aumento do cuidado com os outros e a busca por formas criativas de expressar sua generosidade (Algoe, 2012; Algoe \& Haidt, 2009; Bonne \& De Wall, 2004; Fredrickson, 2002; McCullough et al., 2001; Schwartz, 1967).

Observa-se que há consenso entre os autores de que a gratidão é uma emoção positiva que se expressa no reconhecimento e agradecimento às fontes externas - que podem ser pessoas, coisas inumanas ou Deus (conforme sua prática religiosa). Esse aspecto evidencia o caráter relacional e social da gratidão (Algoe, 2012; Algoe, Fredrickson, \& Gable, 2013; Algoe, Haidt, \& Gable, 2008; Emmons, 2009; McCullough, Tsang, \& Emmons, 2004). Há divergência, porém, quanto à reciprocidade na expressão da gratidão. Bonne e De Wall (2004) e Tsang (2006) defendem que não há sentimento de gratidão por ações não intencionais, já que o beneficiário avaliaria os custos e a intencionalidade do benfeitor, buscando sempre retribuir o favor recebido.

Essa noção de reciprocidade é refutada com base na função moral da gratidão. $\mathrm{O}$ argumento principal é que, mesmo que o beneficiário sinta o desejo de recompensar e valorizar o benfeitor (sua natureza social), estabelece-se a possibilidade da experiência de gratidão justamente pelo beneficiário depender de outrem em uma situação assimétrica, que não lhe permite a reciprocidade. A função moral da gratidão é resultante de afetos ou de qualidades de caráter (também denominadas virtudes) que conduz a ação de se levar o outro em consideração (McCullough et al., 2001; Peterson \& Seligman, 2004). Afetos como empatia, culpa, vergonha ou compaixão, além das qualidades de coragem, justiça e generosidade estão entre as variáveis latentes mais estudadas em relação à gratidão, nessa perspectiva (McCullough et al., 2001; Peterson \& Seligman, 2004).

Estudos no campo da Psicologia Positiva demonstram que pessoas gratas são mais sociáveis e menos narcisistas, corroborando em seus achados que a expressão da gratidão implica em depender de fontes externas para o seu bem-estar. Também há evidências de que vivenciar experiências de agradecimento resulta em respostas mais positivas aos acontecimentos da vida pela lembrança das situações em que se obteve ajuda ou apoio (Donno \& McCullough, 2006; Fredrickson, 1998). O estado psicológico e disposicional de ser grato compõe a memória com lembranças afetivas do que resultou na emoção positiva da gratidão. Seu elemento constitutivo, então, está na disposição psicológica em reconhecer e expressar agradecimento pela ação que lhe trouxe benefício, sem que isso seja similar a qualquer direito adquirido pela fonte externa da ação benevolente (Emmons \& McCullough, 2003; McCullough et al., 2001; Wood, Froh, \& Gerathy, 2010).

$\mathrm{Na}$ presente pesquisa, o construto gratidão foi embasado nos seus elementos constitutivos, para os quais há achados robustos e consenso dos pesquisadores, de modo a valorizar as suas funções social e moral. E, simultaneamente, superar a carência de uma definição ontológica evidente da gratidão. Desse ponto em diante, o construto está assim definido: gratidão é um estado mental positivo de agradecimento por experiências de vida, mesmo que adversas ou de risco, vinculadas à memória das lembranças afetivas que aumentam o bem-estar subjetivo.

Para avaliar a gratidão, há cinco escalas com propriedades psicométricas descritas. A Gratitude Questionnaire seis itens, (McCullough, Emmons, \& Tsang, 2002) e a Escala de Gratitud - 18 itens, (Alarcón, 2014) são medidas unifatoriais do construto, definido como comportamento pró-social. Já o The Gratitude Resentment, and Appreciation - 44 itens (Watkins et al., 2003) a avalia como traço de personalidade. Finalmente, a The Transpersonal Gratitude Scale - 16 itens, (Hlava, Elfers, \& Offringa, 2014) e o El Cuestionario de Gratitud - 20 itens, (Bernabé-Valero, García-Alandete, \& Gallego-Pérez, 2014) é composto por quatro fatores relacionados às fontes externas, emoções interpessoais, reciprocidade e relações sociais. Visto que essas escalas não avaliam o construto que depreende desta revisão teórica, a presente pesquisa teve como 
objetivo desenvolver um instrumento psicométrico para mensurar a gratidão aqui definida, com evidências de validade no Brasil.

Nesta investigação, optou-se ainda por realizar a análise da correlação com a variável latente da esperança disposicional (Pacico et al., 2013) por observar que gratidão e a esperança são construtos com evidências de seu desenvolvimento como preditores de saúde mental. Ambos favorecem o fortalecimento individual e o equilíbrio emocional, aumentando, assim, a capacidade de responder mais satisfatoriamente aos desafios e às adversidades enfrentadas na vida (Alves, 2010; Emmons \& Stern, 2013; Hutz, 2014; Lopez, Floyd, Ulven, \& Snyder, 2000; Lubomirsky, 2008; Magaletta \& Oliver, 1999; Seligman, 2011; Snyder \& Lopes, 2009). Cabe registrar que a esperança disposicional é definida como um estado mental positivo presente em uma tríade formada por objetivo (a busca por algo), rota (possibilidade de caminho para alcançar o objetivo) e agenciamento (motivação para traçar a rota e chegar ao objetivo). $\mathrm{O}$ agente busca atingir seus objetivos ao traçar e percorrer caminhos de execução ou rotas de desenvolvimento diante de situações de vida que se caracterizam como obstáculos, desafios, adversidades ou risco (Snyder Irving, \& Anderson, 1991; Snyder \& Lopez, 2009). Considerando que emoções como a gratidão tendem a influenciar o nível de esperança de forma positiva, impactando o dia a dia (Ouweneel, Le Black, Schaufeli, \& Wijhe, 2012), interessa-nos investigar de forma exploratória essa relação.

\section{Método}

\section{Participantes}

A amostra de conveniência está composta por 762 participantes das cinco regiões brasileiras, com $48 \%$ do Sul, $31 \%$ do Sudeste, $5 \%$ do Centro-Oeste, $12 \%$ do Nordeste, e $4 \%$ do Norte. As idades dos participantes variaram de 18 a 90 anos $(M=41$ anos; $D P=13$ anos), sendo $18 \%$ do sexo masculino. No momento da pesquisa, $43 \%(n=330)$ dos participantes não tinham filhos, enquanto $57 \%(n=432)$ tinham de um a seis filhos. Entre os participantes, $66 \%(n=473)$ afirmou ter algum tipo de prática religiosa. Quanto ao nível de escolaridade, $85 \%(n=649)$ possuíam formação superior completo $(28 \%, n=210)$ ou pós-graduação em nível lacto ou stricto senso $(58 \% ; n=439)$; sendo que $15 \%(n=113)$ afirmaram possuir formação média ou técnica, completa ou incompleta.

\section{Instrumentos}

Escala Brasileira de Gratidão (B-GRAT). A B-GRAT foi desenvolvida com objetivo de avaliar a gratidão na população nacional, definida nesta pesquisa como um estado mental positivo de agradecimento por experiências de vida, mesmo as adversas ou de risco, vinculadas à memória de lembranças afetivas que aumentam o bem-estar subjetivo. Sua versão inicial foi composta por uma pergunta geral (quão grato me sinto hoje?), com escala tipo Likert de cinco pontos (quase nada -1 até muito -5), e 12 itens que elaborados para mensurar a gratidão quanto à: (a) fontes externas de pessoas (sou grato a muitas pessoas), coisas (sou grato por muitas coisas na vida, mesmo quando passei por momentos difíceis) e Deus (sou grato a Deus); (b) situações sociais sobre as quais não tem controle (quando olho para o Brasil não vejo muita coisa para ser grato); (c) estado disposicional (a medida que fico mais velho, sinto-me mais capaz de agradecer as pessoas); e (d) extinção do comportamento (pode passar um longo tempo até que me sinta grato a alguma experiência vivida). Os itens foram mensurados em uma escala tipo Likert para mensurar o grau de concordância dos respondentes, que vai de "totalmente falsa" (1) até "totalmente verdadeira" (5).

Escala de Esperança Disposicional. A Escala foi adaptada da Hope Index Scale (Snyder et al., 1991) e validada para o Brasil com consistência interna e $\alpha=0,80$ (Pacico et al, 2013). O instrumento é composto por 12 itens, em que quatro itens avaliam rotas e outros quatro mensuram agenciamento. Os itens restantes são filtros distratores. A escala Likert de cinco pontos para expressar o grau de concordância com cada afirmativa vai de "totalmente falsa" (1) até "totalmente verdadeira" (5).

\section{Procedimentos}

Foram elaborados 13 itens para mensurar a gratidão. Esses itens foram desenvolvidos com base em uma extensa revisão teórica. A validação de conteúdo dos itens da escala de gratidão desenvolvida foi realizada por três juízes, com conhecimentos em psicometria e publicações no campo da Psicologia Positiva. Os juízes avaliaram o instrumento quanto à relevância teórica dos itens para o constructo (Hutz, 2015).

A versão preliminar com as sugestões de ajustes dos juízes foi avaliada por três grupos focais, divididos de acordo com nível de escolaridade: participantes que concluíram o ensino médio $(n=4)$; participantes que concluíram o ensino superior ou estão cursando sua graduação $(n=3)$; participantes que estavam cursando ou concluíram uma pós-graduação $(n=3)$ no momento da pesquisa. Foi solicitado nos grupos focais que os participantes lessem todos os itens, relatassem sua compreensão de cada um e indicassem se havia a necessidade de incluir novos itens ou aspectos importantes para medir gratidão em brasileiros. Os participantes com diferentes níveis de escolaridade apresentaram uma compreensão adequada da escala e dos itens apresentados. Os três grupos apresentaram relatos sobre as suas experiências de gratidão como vivências positivas, por meio das suas memórias sobre os acontecimentos, mesmo quando não observavam a benevolência nas pessoas ou nas situações. Foi observado que os participantes de menor escolaridade enfatizaram sua 
gratidão a Deus, sem relacionar a uma prática religiosa específica, por situações críticas em que pensavam que nunca iriam se sentir gratos. Em razão disso, a palavra Deus permaneceu nos itens, ao invés do termo "ação divina". Os grupos focais ocorreram até a saturação teórica, sem que houvesse outras alterações ou inclusão de novos itens.

A coleta de dados foi on-line, realizada em redes sociais, por meio da ferramenta SurveyMonkey, entre fevereiro e março de 2019. Ao serem convidados a colaborar com o estudo, todos os participantes eram informados sobre os objetivos da pesquisa, sendo que apenas aqueles que concordavam com o Termo de Consentimento Livre Esclarecido - aprovado pelo CEP/UFCSPA, parecer número 2.343.078 - obtiveram acesso aos instrumentos da pesquisa.

\section{Análise de dados}

A análise da dimensionalidade da Escala de Gratidão foi realizada utilizando dois critérios de extração: o pressuposto teórico de que a gratidão se constitui como um construto único e a análise paralela (Hayton, Allen, \& Scarpello, 2004). Os dois critérios indicaram que a Escala de Gratidão apresentava uma estrutura unifatorial.

A dimensionalidade da escala de gratidão foi avaliada por meio da análise fatorial confirmatória (AFC). No modelo testado todos os itens carregavam em apenas um fator. A avaliação do modelo foi realizada utilizando o método de estimação Weighted Least Squares Mean and Variance-Adjusted (WLSMV), por esse ser um método de estimação robusto para dados ordinais (Muthén \& Muthén, 2010). Os índices de ajuste foram os seguintes: Comparative Fit Index (CFI>0,90); Tucker Lewis Index (TLI>0,90); e Root Mean Square Error of Approximation (RMSEA $<0,08$, com intervalo de confiança de $90 \%$ não excedendo a 0,10) (Brown, 2006). Os índices de modificação (IM) com valores acima de 50,00 foram avaliados, para identificar fontes de problemas na especificação do modelo (Brown, 2006).

Além da dimensionalidade da escala, as cargas fatoriais e os thresholds dos itens foram avaliados. Esses indicadores foram analisados para investigar em profundidade a precisão dos itens, por meio das cargas fatoriais e os limiares de dificuldade dos itens, por meio dos thresholds. Após a definição da estrutura da Escala de Gratidão foram investigados os índices de consistência interna da escala e avaliados por meio do coeficiente de fidedignidade composta $(f c)$ e o alfa de Crobanch $(\alpha)$.

Para avaliar a relação entre gratidão e as características sociodemográficas, foram testadas as diferenças entre os escores de homens e mulheres, escolaridade (superior/pós-graduado ou técnico) e praticantes ou não de alguma religião. Foram realizadas análises do teste $t$ para verificar as diferenças entre esses grupos. Além disso, foi calculado o tamanho de efeito $d$ de Cohen para investigar a importância prática dos resultados.
Finalmente, foram investigadas as relações entre gratidão, esperança, idade e número de filhos. Destaca-se que as análises de comparação foram realizadas utilizando os escores fatoriais de gratidão e esperança. Esses escores fatoriais foram obtidos por meio de AFC, realizada com método de estimação WLSMV. Devido ao expressivo tamanho da amostra, o nível de significância de todas as análises inferenciais (equações estruturais e comparação de média) foi estabelecido em $0,001(p<0,001)$. Já o teste das associações entre essas variáveis foi realizado por meio da modelagem de equações estruturais. Para avaliar o efeito prático dessas associações, o tamanho de efeito das relações foi calculado por meio do $r^{2}$.

\section{Resultados}

A análise fatorial exploratória indicou a retirada dos itens relacionados à intensidade da gratidão (um item geral), às situações fora do controle do respondente (um item) e à extinção do comportamento de gratidão (quatro itens). Em razão disso, seis itens foram excluídos da B-GRAT e foi testado um modelo no qual os sete itens restantes carregaram em uma única dimensão. O pressuposto teórico e os resultados da análise paralela indicaram que a estrutura unidimensional é a mais adequada para a escala de gratidão. Nesse modelo, todos os itens que possuíam carga fatorial superior a 0,60 carregaram no fator geral. Os índices de ajuste desse modelo foram satisfatórios, mas os níveis de resíduos estavam acima do valor considerado adequado (Ver Tabela 1).

A fim de melhorar os valores dos resíduos, os índices de modificação do primeiro modelo foram analisados e indicaram que os resíduos dos itens 6 e 7 poderiam ser correlacionados. No segundo modelo testado, a correlação do par de itens 6 e 7 foi incluída, os índices de ajuste foram excelentes e todos os itens obtiveram carga fatorial acima de 0,60 (Ver Tabela 1). Além disso, foi observado que os valores de consistência interna da Escala de Gratidão foram satisfatórios $(f c=0,99 ; \alpha=0,84)$ (Ver Tabela 1).

A análise das cargas fatoriais demonstra que os itens apresentam precisão, tendo em vista que as cargas fatoriais foram altas, variando de 0,67 a 0,86 (Ver Tabela 1). Com relação aos thresholds, observa-se que há uma diminuição gradual das dificuldades de resposta ao longo da escala intervalar, de modo que, no processo de resposta do mesmo item, a dificuldade é menor conforme a opção de resposta se localiza mais próxima da alternativa "Totalmente verdadeira". Entre os sete itens que compõe a escala, o item 5 "Sou grato(a) a muitas pessoas." foi o que apresentou menor dificuldade de resposta e menor abrangência do construto gratidão. Já o item 4 "Quando olho para o mundo, vejo muita coisa para ser grato(a)." pode ser avaliado como o item com maior dificuldade na resposta e maior abrangência do construto. 
Tabela 1

Análise Fatorial Confirmatória da B-GRAT

\begin{tabular}{|c|c|c|c|c|c|c|c|c|c|c|}
\hline \multirow{2}{*}{ Itens } & \multicolumn{5}{|c|}{ Modelo 1} & \multicolumn{5}{|c|}{ Modelo 2} \\
\hline & CF & $\delta j 1$ & 8j2 & 8j3 & 8j4 & CF & \multicolumn{4}{|c|}{ Thresholds } \\
\hline $\begin{array}{l}\text { 1. Eu sou grato(a) por muitas coisas } \\
\text { na vida, mesmo quando passei por } \\
\text { momentos difíceis. }\end{array}$ & $0,68^{*}$ & $-2,35$ & $-1,57$ & $-0,85$ & $-0,01$ & $0,71^{*}$ & $\delta j 1$ & $\delta \mathrm{j} 2$ & $\delta j 3$ & $\delta j 4$ \\
\hline $\begin{array}{l}\text { 2. Se tivesse que listar tudo pelo } \\
\text { que sou grato(a), essa seria uma } \\
\text { lista muito longa. }\end{array}$ & $0,83^{*}$ & $-2,35$ & $-1,60$ & $-0,83$ & $-0,15$ & $0,86^{*}$ & $-2,35$ & $-1,57$ & $-0,85$ & $-0,01$ \\
\hline $\begin{array}{l}\text { 3. Quando olho para o mundo vejo } \\
\text { muita coisa para ser grato(a). }\end{array}$ & $0,78^{*}$ & $-2,5$ & $-1,6$ & $-0,77$ & $-0,04$ & $0,81^{*}$ & $-2,35$ & $-1,60$ & $-0,83$ & $-0,15$ \\
\hline 4. Sou grato(a) a muitas pessoas. & $0,71^{*}$ & $-2,78$ & $-1,53$ & $-0,77$ & $-0,07$ & $0,73^{*}$ & $-2,55$ & $-1,60$ & $-0,77$ & $-0,04$ \\
\hline 5. Sou grato(a) a Deus. & $0,63^{*}$ & $-1,51$ & $-1,20$ & $-0,88$ & $-0,52$ & $0,65^{*}$ & $-2,78$ & $-1,53$ & $-0,77$ & $-0,07$ \\
\hline $\begin{array}{l}\text { 6. À medida que fico mais velho, } \\
\text { sinto-me mais capaz de agradecer } \\
\text { as pessoas. }\end{array}$ & $0,81^{*}$ & $-2,40$ & $-1,73$ & $-1,03$ & $-0,25$ & $0,67^{*}$ & $-1,51$ & $-1,20$ & $-0,88$ & $-0,52$ \\
\hline \multirow{2}{*}{$\begin{array}{l}\text { 7. À medida que fico mais velho, } \\
\text { sinto-me mais capaz de agradecer } \\
\text { as situações que têm feito parte da } \\
\text { minha história de vida. }\end{array}$} & $0,85^{*}$ & $-2,40$ & $-1,78$ & $-1,17$ & $-0,32$ & $0,71^{*}$ & $-2,40$ & $-1,735$ & $-1,03$ & $-0,25$ \\
\hline & \multicolumn{3}{|c|}{$\chi^{2}(g l)$} & CFI & TLI & \multicolumn{3}{|c|}{ RMSEA (90\% CI) } & FC & $\alpha$ \\
\hline Modelo 1 & \multicolumn{3}{|c|}{$228,50^{*}(14)$} & 0,95 & 0,93 & \multicolumn{3}{|c|}{$0,143(0,127-0,160)$} & 0,99 & 0,84 \\
\hline Modelo 2 (e6 - e7) & \multicolumn{3}{|c|}{$29,77^{*}(13)$} & 0,99 & 0,99 & 0,041 & 0,022 & $0,061)$ & 0,99 & 0,84 \\
\hline
\end{tabular}

Nota: ${ }^{*} p<0,001, n=750, \mathrm{CF}=$ Carga Fatorial do Item

Por outro lado, os escores de gratidão apresentaram relações positivas de alta magnitude com os níveis de esperança dos participantes. Foi observado também que a gratidão esteve positivamente associada à idade e ao número de filhos, sendo que essa associação apresentou magnitude média (Ver Tabela 2). A variância explicada da relação entre gratidão e esperança foi de $r^{2}=38 \%$, o que confirma a associação entre esses dois estados mentais positivos, que se caracterizam como uma variável disposicional e latente do comportamento humano. Já a idade dos participantes apresenta variância compartilhada de $r^{2}=14 \%$ com gratidão e de $r^{2}=31 \%$ com o número de filhos; o que significa que há desenvolvi- mento da gratidão ao longo da vida, especialmente para quem tem filhos.

Também foi avaliado se os participantes apresentavam diferenças devido às suas características sociodemográficas. Os resultados indicam que as mulheres, os praticantes religiosos e as pessoas com maior nível de escolaridade apresentam escores de gratidão mais altos. $\mathrm{Na}$ Tabela 3, pode ser observado que os tamanhos de efeito das diferenças entre os participantes do sexo masculino e feminino, e entre aqueles que praticam uma religião ou não tem prática religiosa, apresentam alta magnitude. Já entre os especialistas e técnicos, observa-se que as diferenças apresentam magnitude média.

Tabela 2

Relações entre Gratidão, Esperança, Idade e Número de Filhos dos Participantes

\begin{tabular}{lccc}
\hline & 1 & 2 & 3 \\
\hline 1. Gratidão & & & \\
2. Esperança & $0,62^{*}$ & & \\
3. Idade & $0,37^{*}$ & $0,26^{*}$ & $0,56^{*}$ \\
4. Filhos & $0,30^{*}$ & $0,20^{*}$ & \\
\hline
\end{tabular}

Nota. ${ }^{*} p<0,001$ 
Tabela 3

Diferenças das Médias dos Escores de Gratidão para Sexo, Escolaridade e Prática Religiosa (n=750)

\begin{tabular}{lccc}
\hline & Gratidão M (DP) & $t(g l)$ & \\
\hline Sexo & & $4,60^{*}(748)$ & \\
Masculino & $4,0(0,8)$ & & \\
Feminino & $4,4(0,6)$ & & \\
& & & \\
Escolaridade & & $5,30^{*}(748)$ & \\
Técnico & $4,2(0,7)$ & & \\
Especialista & $4,4(0,6)$ & & \\
Religião & & $-6,30 *(748)$ & \\
Não praticante & & & 0,50 \\
Praticante & $4,1(0,7)$ & & \\
\hline
\end{tabular}

Nota. ${ }^{*} p<0,001$

\section{Discussão}

O objetivo do presente estudo foi desenvolver a Escala Brasileira de Gratidão (B-GRAT) para mensurar esse construto no Brasil. Para tanto, partiu-se dos estudos na área para elaborar a definição do conceito de gratidão a ser testado nessa pesquisa. A escala com sete itens apresentou evidência de validade adequada para seu uso. Conclui-se que a B-GRAT apresenta a estrutura esperada em termos de consistência interna e validade fatorial. Entre as contribuições dessa pesquisa, destaca-se o avanço teórico na definição conceitual da gratidão, o que permitirá verificar sua relação com outras variáveis latentes do comportamento humano de forma acurada e consistente. O que permitirá aos pesquisadores aprofundar a compreensão de sua dinâmica e seu processo de desenvolvimento, testando suas associações com variáveis que a literatura científica aponta como importantes.

A relação entre esperança e gratidão foi demonstrada neste estudo. Sugere-se investigações que contribuam para avançar teoricamente nesse tema, considerando o possível papel da esperança como mediadora da relação da gratidão com outras variáveis em saúde mental. Os estudos em esperança disposicional demonstram que o agente humano é capaz de planejar, explorar e executar rotas que conduzem ao bem-estar subjetivo. Já pesquisas em gratidão indicam que o reconhecimento e a valorização da ação benevolente nas situações em que se está dependente de fontes externas é um fator importante para a satisfação de vida e para as emoções positivas que podem ser associar. Sua relação, já apontado nos estudos da área, com as variáveis latentes de bem-estar faz com que a expressão da gratidão contribua para a saúde mental das pessoas. Os achados, de modo geral, indicam o papel da gratidão como fator de proteção e prevenção de transtornos de ansiedade e depressão, além de outros. Estudos futuros nessa linha serão importantes indicadores de preditores e mediadores da saúde mental dos brasileiros.

Foram limitações desta pesquisa a amostra predominantemente do sexo feminino e com concentração nas regiões Sul e Sudeste do Brasil. O que sugere que novos estudos devem ser realizados para verificação das normas para a população brasileira, além de pesquisas para testar a validade discriminante e a estabilidade fatorial da B-GRAT. Destaca-se, porém, que os dados apresentados indicam a importância de compreender aspectos relacionados às diferenças entre homens e mulheres, religiosos praticantes e não religiosos e pessoas com diferentes escolaridades. Entende-se que tais aspectos se apresentam como relevantes para compreensão dos aspectos constitutivos da gratidão.

Por outro lado, cabe ressaltar que o conceito de gratidão proposto neste artigo o relaciona ao desenvolvimento de recursos pessoais (memórias e lembranças afetivas, experiências de agradecimento, afetos e sentimentos) com potencial de conduzir ao bem-estar subjetivo e à saúde mental. Faz-se necessário aprofundar os conhecimentos científicos sobre como e por que algumas pessoas, com determinadas características sociodemográficas, desenvolvem a gratidão. Pesquisas que investiguem o papel da personalidade nesse processo, dentre outros constructos, podem contribuir para aprimorar esse entendimento.

\section{Referências}

Alarcón, R. (2014). Construcción y valores psicométricos de una escala para medir la gratitud. Acta de Investigación Psicológica, 4(2), $1520-1534$. doi: 10.1016/S2007-4719(14)70391-6 
Algoe, S. B. (2012). Find, remind, and bind: The functions of gratitude in everyday relationships. Social and Personality Psychology Compass, 6(6), 455-469. doi: 10.1111/j.1751-9004.2012.00439.x

Algoe, S. B., \& Haidt, J. (2009). Witnessing excellence in action: The "other-praising" emotions of elevation, gratitude, and admiration. The journal of positive psychology, 4(2), 105-127. doi: 10.1080/17439760802650519

Algoe, S. B., Fredrickson, B. L., \& Gable, S. L. (2013). The social functions of the emotion of gratitude via expression. Emotion, 13(4), 605609. doi: 10.1037/a0032701

Algoe, S. B., Haidt, J., \& Gable, S. L. (2008). Beyond reciprocity: Gratitude and relationships in everyday life. Emotion, 8(3), 425-429. doi: 10.1037/1528-3542.8.3.425

Alves, A. F. A. M. V. (2010). Gratidão: um estudo longitudinal sobre o impacto pessoal e relacional. (Dissertação de Mestrado), Universidade de Lisboa, Lisboa, Portugal. Recuperado de https://repositorio.ul.pt/bitstream/10451/3050/1/ulfp037537_tm.pdf

Bernabé-Valero, G., García-Alandete, J., \& Gallego-Pérez, J. F. (2014). Construcción de un cuestionario para la evaluación de la gratitud: El Cuestionario de Gratitud-20 ítems (G-20). Anales de Psicología, 30(1), 278-286. doi:10.6018/analesps.30.1.135511

Bonnie, K. E. \& de Wall, F. B. M. (2004). Primate social reciprocity and the origin of gratitude. In R. A. Emmons \& M. E. McCullough (Orgs.), The psychology of gratitude (pp. 213-229). New York: Oxford University Press.

Bono, G. \& Mccullough, M. E. (2006). Positive responses to benefit and harm: Bringing forgiveness and gratitude into cognitive psychotherapy. Journal of Cognitive Psychotherapy, 20(2):147-158. doi: 10.1891/088983906780639835

Brown, T. A. (2006). Confirmatory factor analysis for applied research. NY: Guilford.

Emmons, R. A., \& Mccullough, M. E. (2003). Counting blessings versus burdens: An experimental investigation of gratitude and subjective well-being in daily life. Journal of Personality and Social Psychology, 84(2), 377-389. doi: 10.1037/0022-3514.84.2.377

Emmons, R. A., \& Mishra, A. (2011). Why gratitude enhances well-being: What we know, what we need to know. Em K. Sheldon, T. B. Kashdan, \& M. Steger (Eds.). Designing positive psychology: Taking stock and moving forward (pp. 248-262). New York, US: Oxford University Press. doi:10.1093/acprof:oso/9780195373585.003.0016

Emmons, R. A., \& Stern, R. (2013). Gratitude as a psychotherapeutic intervention. Journal of Clinical Psychology, 69(8), 846-855. doi: 10.1002/ jclp.22020.

Frederickson, B. L. (1998). What good are positive emotions? Review of General Psychology, 2(3): 300-319. doi: 10.1037/1089-2680.2.3.300

Frederickson, B. L. (2002). Positive emotions. Em C. R. Snyder \& S. J. Lopez (Eds.), Handbook of positive psychology (pp. 120-134). New York: Oxford University Press.

Fredrickson, B. L. (2013). Positive Emotions Broaden and Build. Em P. Devine, \& A. Plant (Eds.), Advances in Experimental Social Psychology (pp. 1-53). Burlington: Academic Press. doi:10.1016/B978-0-12-407236-7.00001-2

Hayton, J. C.; Allen, D. G. \& Scarpello, V. (2004). Factor Retention Decisions in Exploratory Factor Analysis: A Tutorial on Parallel Analysis. Organizational Research Methods, 7(2):191-205. doi:10.1177/1094428104263675

Hlava, P., Elfers, J., \& Offringa, R. (2014). A transcendent view of gratitude: The transpersonal gratitude scale. International Journal of Transpersonal Studies, 33(1), 1-14. doi: 10.24972/ijts.2014.33.1.1

Hutz, C. S. (2014). Avaliação em psicologia positiva. Porto Alegre: Artmed.

Hutz, C. S. (2015). Psicometria. Porto Alegre: Artmed.

Lopez, S. J., Floyd, K. R., Ulven, J. C., \& Snyder, C. R. (2000). Hope Therapy: Helping clients build a house of hope. Em: Snyder, C. R. (Org). Handbook of hope. Theory, Measures \& Applications (pp. 123-150). NY: Elsevier. doi:10.1016/B978-012654050-5/50009-9

Lyubomirsky, S. (2008). A ciência da felicidade: Como atingir a felicidade real e duradoura. Rio de Janeiro: Elsevier.

Magaletta, P. \& Oliver, J. (1999). The hope construct, will, and ways: Their relations with self-efficacy, optimism, and general well-being. Journal of clinical psychology, 55(5), 539-551. doi:10.1002/(SICI)1097-4679(199905)55:5<539::AID-JCLP2>3.0.CO;2-G

Mccullough, M., Kilpatrick, S., Emmons, R., \& Larson, D. (2001). Is Gratitude a Moral Affect? Psychological bulletin, 127(2) 249-66. doi: 10.1037//0033-2909.127.2.249

McCullough, M. E., Emmons, R. A., \& Tsang, J. A. (2002). The grateful: A conceptual and empirical topography. Journal of Personality and Social Psychology. 82(1), 112-127. doi: 10.1037/0022-3514.82.1.112

McCullough, M. E., Tsang, J. A. \& Emmons, R. A. (2004). Gratitude in intermediate affective terrain: Links of grateful moods to individual differences and daily emotional experience. Journal of Personality and Social Psychology, 86(2), 295-309. doi: 10.1037/00223514.86.2.295

Muthén, L. F., Muthén B. O. (2010). Mplus user's guide: Mplus statistical analysis with latent variables. (6 $6^{\mathrm{a}}$ Ed). Los Angeles: Muthén \& Muthén.

Ouweneel, E., Le Blanc, P., Schaufeli, W. B., \& Van Wijhe, C. (2012). Good morning, good day: A diary study on positive emotions, hope, and work engagement. Human Relations, 65(9)1129-1154. doi: 10.1177/0018726711429382

Pacico, J. C., Zanon, C., Bastianello, M. R., \& Hutz, C. S. (2013). Adaptation and validation of the Brazilian version of the Hope Index. Psicologia: Reflexão e Crítica, 24(4), 666-670. doi: 10.1590/S0102-79722011000400006

Peterson, C., \& Seligman, M. E. P. (2004). Character Stregnths and Virtues: A handbook and classification. New York: Oxford University Press, American Psychology Association.

Pieta, M. A. M., \& Freitas, L. B. L. (2009). Sobre a gratidão. Arquivos Brasileiros de Psicologia, 61(1), 100-108. Recuperado de http://pepsic. bvsalud.org/scielo.php?script $=$ sci_arttext\&pid $=$ S1809-52672009000100010\&lng $=$ pt\&tlng $=$ pt

Salvador-Ferrer, C. (2017). The relationship between gratitude and life satisfaction in a sample of Spanish university students: The moderation role of gender. Anales de Psicología, 33(1), 114-119. doi: 10.6018/analesps.33.1.226671

Sapienza, G., \& Pedromônico, M. R. M. (2005). Risco, proteção e resiliência no desenvolvimento da criança e do adolescente. Psicologia em Estudo, 10(2): 209-216. doi: 1590/S1413-73722005000200007

Schwartz, B. (1967). The Social Psychology of the Gift. American journal of sociology, 73(1) 1-11. doi: 10.1086/224432.

Seligman, M. E. P. (2011). Florescer: Uma nova compreensão sobre a natureza da felicidade e do bem-estar. Rio de Janeiro: Objetiva.

Snyder, C. R., \& Lopez, S. J. (2009). Psicologia Positiva: uma abordagem científica e prática das qualidades humanas. Porto Alegre: Artmed.

Snyder, C. R., Irving, L., \& Anderson, J. R. (1991). Hope and health: Measuring the will and the ways. Em C. R. Snyder, \& D. R. Forsyth (Eds.), Handbook of social and clinical psychology: The health perspective (pp. 285-305). Elmsford, NY: Pergamon Press.

Tsang, J. (2006). Gratitude and prosocial behaviour: An experimental test of gratitude. Cognition and Emotion, 20(1),138-148. doi:10.1080/02699930500172341 
Watkins, P. C., Woodward, K., Stone, T., \& Kolts, R. L. (2003). Gratitude and happiness: Development of a measure of gratitude, and relationships with subjective wellbeing. Social Behavior and Personality, 31(5), 431-451. doi: https://doi.org/10.2224/sbp.2003.31.5.431

Wood, A. M., Froh, J. J., \& Gerathy, A. W. A. (2010). Gratitude and well-being: A review and theoretical integration. Clinical Psychology Review, 30(2010), 890-905. doi: 10.1016/j.cpr.2010.03.005

\section{Sobre os autores}

Ana Claudia Souza Vazquez é psicóloga pela Universidade Federal do Rio de Janeiro (UFRJ) e doutora em administração pela Universidade Federal do Rio Grande do Sul (UFRGS), Atualmente é pró-reitora de gestão com pessoas e atua no Programa de Pós-Graduação Psicologia e Saúde, na Universidade Federal de Ciências da Saúde de Porto Alegre (UFCSPA).

Joice Franciele Friedrich Almansa é psicóloga pela Universidade do Sul de Santa Catarina (Unisul) e especialista em Psicodrama pela Instituição Viver Psicologia: Psicodrama. Atualmente é mestranda do Programa de Pós-Graduação em Psicologia e Saúde na Universidade Federal de Ciências da Saúde de Porto Alegre (UFCSPA).

Clarissa Pinto Pizarro de Freitas é doutora em Psicologia pela Universidade Federal do Rio Grande do Sul (UFRGS). Atualmente é professora da Pós-Graduação de Psicologia na Universidade Salgado de Oliveira (UNIVERSO).

Claudio Simon Hutz é psicólogo, mestre e PhD pela University of Iowa (USA) e professor no PPG de Psicologia da UFRGS. 